\title{
Aplicação do pressure ulcer knowledge test em enfermeiros de um hospital de atenção secundária - estudo transversal
}

\section{Application of pressure ulcer knowledge test in nurses at a secon- dary care hospital - cross-sectional study}

\author{
Paula Arquioli Adriani' • André Oliveira Paggiaro² • Marcus Castro Ferreira ${ }^{3}$ \\ Viviane Fernandes de Carvalho ${ }^{4}$
}

\begin{abstract}
RESUMO
Objetivo: identificar o nível de conhecimento de enfermeiros sobre a avaliação, prevenção e classificação de lesões por pressão em um hospital do Rio de Janeiro. Métodos: Trata-se de um estudo do quantitativo com delineamento descritivo-exploratório. Os dados foram coletados sem a realização de qualquer tipo de orientação ou treinamento prévio dos profissionais sobre lesão por pressão (LPP). Para seu desenvolvimento, foi utilizado o Pressure Ulcer Pieper Knowledge Test (PUKT), que é composto de $4 \mathrm{I}$ questões sobre avaliação, classificação e prevenção de lesões por pressão. Destas, 8 questões referem-se a avaliação e classificação da LPP e 33 questões sobre prevenção. Resultados: Participaram 102 enfermeiros, sendo 7I do sexo feminino e 31 do sexo masculino. A predominância de idade dos participantes foi de 30 a 39 anos. Quanto ao tempo de formado a maioria possui entre 2 a 5 anos de conclusão de curso e formação em Latu senso. Em relação aos resultados globais do teste, $70 \%$ (68.63\%) dos enfermeiros acertaram menos de $70 \%$ das questões, indicando um déficit de conhecimento na área. Conclusão: Diante dos resultados, identifica-se um déficit no conhecimento sobre a avaliação, classificação e prevenção de LPP, indicando a necessidade da educação permanente para atualização dos profissionais de enfermagem.
\end{abstract}

Palavras Chave: Enfermagem; conhecimento; lesão por pressão; classificação.

\begin{abstract}
Objective: to identify the level of nurses' knowledge on evaluation, prevention and classification of pressure ulcers in a hospital in Rio de Janeiro. Methods: This is a quantitative study with a descriptive-exploratory design. The data were collected without conducting any kind of orientation or training on pressure ulcer (LPP). For its development, the Pressure Ulcer Pieper Knowledge Test (PUKT) was used, which is composed of 4 I questions about evaluation, classification and prevention of Pressure Ulcers. Of these, 8 questions refer to the evaluation and classification of the UPP and 33 questions are about prevention. Results: 102 nurses participated, 71 female and 31 male. The participants' predominance of age was 30 to 39 years. As for the time of training, the majority has between 2 to 5 years of conclusion of course and the majority has formation in Latu senso. Regarding the overall results of the test, $70 \%$ (68.63\%) of nurses scored less than $70 \%$ of the questions, indicating a lack of knowledge in the area. Conclusion: In view of the results, a deficit in the knowledge about the evaluation, classification and prevention of LPP is identified, indicating the need for permanent education to update and improve nursing professionals.
\end{abstract}

Keywords: Nursing; Knowledge; Pressure Ulcer; Classification. 


\section{INTRODUÇÃO}

As lesões por pressão (LPP) são uma das principais complicações da hospitalização prolongada, especialmente em situações de má nutrição, aumento da umidade na pele (por exemplo, incontinência urinária ou fecal), pressão prolongada e comprometimento de receptores sensoriais. Estas feridas aumentam o custo da internação, da morbidade e mortalidade dos pacientes, e desempenham papel significativo na propagação da infecção nos ambientes clínicos ${ }^{(1-2)}$.

Em geral, profissionais e leigos fazem uso de termos incorretos para a LPP como o termo "Escara" que consiste em um estágio pelo qual a LPP pode passar. Neste estágio há formação de tecido necrótico que pode existir sobre a úlcera e este se denomina escara, logo, uma lesão por pressão pode estar recoberta ou não por escara. Outro termo empregado, mas incorreto, é "Úlcera de decúbito", que se analisada dentro de sua raiz, vê-se que a palavra "decúbito", é originaria do latim "decumbere", e significa "deitado" e sua ocorrência não incide somente em locais onde há pressão exercida sobre proeminências ósseas com o paciente deitado (1-3-4).

As úlceras por pressão têm prevalência e incidência elevadas em pacientes hospitalizados e/ou acamados, e os estudos mostram que seu desenvolvimento pode ocorrer em um período de 24 horas com 5 dias para sua manifestação ${ }^{(5)}$. Em estudo transversal realizado nos Estados Unidos, identificou-se uma prevalência de LPP em hospitais de 13,5\% ${ }^{(66)}$.Já em estudo realizado na Espanha, aponta que a população hospitalizada mostra uma variabilidade de 3,5\% a $29 \%$, com uma incidência global de $8 \%$ para desenvolver a LPP ${ }^{(7)}$.

Considerando a etiologia das LPP, a pressão nos tecidos é examinada em relação a três fatores: intensidade da pressão, duração da pressão e tolerância tecidual ${ }^{(8)}$.

A prevenção das LPP focaliza questões que devem estar diretamente relacionadas às medidas adotadas pela equipe multiprofissional, sendo priorizada principalmente pela ação e intervenção do enfermeiro, que propõe e auxilia no conhecimento e na aplicação de programas de prevenção e as medidas interventivas adotadas pela equipe. Em geral, a prevenção é menos dispendiosa que seu tratamento e quando se aprende a reconhecer os pacientes em risco, à implantação de medidas apropriadas no seu manejo tornase mais adequada e eficaz, reduzindo os fatores predisponentes e consequentemente reduzindo os custos ${ }^{(9)}$.

A implementação de estratégias de prevenção da formação de feridas depende de uma adequada avaliação e classificação das LPPs, entretanto, muitas vezes, os profissionais envolvidos neste trabalho demonstram desconhecimento ou desatualização sobre o assunto, prejudicando - estabelecimento de políticas hospitalares preventivas. Recentemente, em um hospital escola brasileiro da cidade de Manaus-AM, um estudo avaliou o conhecimento da equipe da equipe de enfermagem sobre a prevenção de LPP, detectando um déficit de conhecimento tanto entre enfermeiros como técnicos de enfermagem ${ }^{(10)}$.

Diante da função de liderança e grande influência dos enfermeiros na elaboração de planos de prevenção de LPPs nos hospitais brasileiros, o objetivo deste estudo foi identificar o nível de conhecimento destes profissionais sobre a prevenção de úlceras por pressão em um hospital brasileiro do estado do Rio de Janeiro.

\section{MÉTODO}

Trata-se de um estudo do tipo quantitativo com delineamento descritivo-exploratório com análise de dados, implantado a partir da utilização do instrumento validado "PUKT", que avalia o nível de conhecimento de enfermeiros sobre prevenção, avaliação e classificação de LPP.

A pesquisa foi realizada em um hospital público estadual de grande porte da cidade do Rio de Janeiro, que possui 267 leitos e 225 enfermeiros, e ocorreu após a autorização da Comissão de Ética para Análise de Projetos de Pesquisa (CAPPESQ) do Hospital das Clínicas da Faculdade de Medicina da Universidade de São Paulo, sob o número de processo: 0223/08, e da convalidação da diretora de enfermagem do Hospital Pesquisado em respeito à resolução 466/2012 do Conselho Nacional de Saúde (CNS).

A amostra foi realizada por conveniência considerando a aceitação voluntária e individual da população em participar da pesquisa, favorecendo a captação das ideias gerais e da identificação dos aspectos críticos da pesquisa, bem como a presença dos mesmos no dia e horário da coleta.

Como critérios de inclusão para o estudo, foram considerados funcionários registrados na instituição como Enfermeiro com tempo mínimo de um ano de serviços prestados e disponibilidade para participar do estudo. Foram excluídos do estudo funcionários que atuavam nas unidades de pediatria, em razão da baixa incidência de LPP nesta unidade, e enfermeiros em período de férias, folga ou licença médica.

A coleta ocorreu entre maio a junho de 20I4. Inicialmente aceitaram participar da pesquisa 125 enfermeiros, porém após a aplicação dos critérios de inclusão e exclusão, a amostra final foi composta por 102 enfermeiros.

Os selecionados receberam um envelope contendo uma cópia do questionário e duas cópias do TCLE. O enfermeiro responsável pela aplicação ficou aguardando junto ao enfermeiro entrevistado até a finalização do preenchimento do questionário e do TCLE, sendo que a abordagem ocorreu exclusivamente em suas unidades e horários de trabalho. Os participantes foram orientados sobre a importância de responder o questionário de forma honesta salientando que as respostas não teriam nenhum caráter punitivo, e sim de pesquisa.

Após a assinatura do Termo de Consentimento Livre 
e Esclarecido (TCLE), aplicou-se o instrumento de coleta composto por dados sócios demográficos dos participantes, acrescido de questões referentes ao tempo de formação, tempo de atuação na instituição, participação em atividades de pesquisa durante a graduação, formação em Lato sensu, formação em Stricto sensu, participação em encontros, jornadas, simpósios, congressos, grupos/comissões de estudo na instituição, atividades educacionais, leitura de revistas científicas e pesquisa por meio da internet.

A seguir, aplicou-se o "PUKT" validado no Brasil pela Dra. Maria Helena Larcher Caliri, do Hospital das Clínicas de Ribeirão Preto (II) sendo composto de 41 questões, 8 questões sobre avaliação e classificação da LPP e 33 sobre prevenção da LPP, as quais podem ser respondidas de forma objetiva, em verdadeiras, falsas ou ainda, como "não sei" permitindo ser tabulado pelo número de respostas corretas. As questões foram embasadas no questionário da Professora Bárbara Pieper, da Wayne State University, coautora do Pieper's "Pressure Ulcer Knowledge Test. (PUKT) "(12).

A análise e interpretação dos dados deu-se por meio da construção do banco de dados no programa do Excel $2010 \mathrm{e}$ SPSS (Statistical Package for the Social Sciences) versão 22, seguidos do uso de gráficos e tabelas em uma análise quantitativa a fim de caracterizar os resultados obtidos do questionário aplicado aos enfermeiros, que analisou os números absolutos e relativos do antes e o depois do processo educativo.

Adotou-se neste estudo o modelo de avaliação do estudo original de Pieper ${ }^{(13)}$, onde cada resposta correta recebeu o valor I (um) e as respostas incorretas, não respondidas ou respondidas como não sabe como valor 0 (zero).A pontuação total no teste de conhecimento foi à soma de todas as respostas corretas. No estudo original, esperava-se que os participantes atingissem $90 \%$ ou mais de acertos para que o conhecimento fosse considerado adequado. Neste estudo, considerou-se como escore de conhecimento a pontuação de 100 a $80 \%$ como ótimo; de $79 \%$ a $59 \%$ como moderado e abaixo de $59 \%$ como baixo, dando segmento ao teste inicial.

\section{RESULTADOS}

A tabela I representa a distribuição dos participantes diante da caracterização sócio demográfica da amostra quanto ao sexo, idade, tempo de formação e tempo de atuação na Instituição de Saúde com 102 (n= 102) enfermeiros que contemplaram os critérios de inclusão e exclusão da pesquisa.

Dos 102 enfermeiros participantes, a predominância foi do sexo feminino, 7 I (69,6\%). Quanto à idade dos participantes, os resultados obtidos foram com uma média de 35,6 anos, sendo o valor mínimo de 23 anos e o valor máximo foi de $6 \mathrm{I}$ anos, estando a maior população distribuída entre 60 enfermeiros $(58,3 \%)$ com idade entre 30 e 39 anos e I (I,2\%) com $6 I$ anos de idade.

A tabela 2 representa a distribuição dos participantes diante da caracterização sócio demográfica da amostra transversal da pesquisa $(n=102)$.

TABELA 1 - Distribuição dos participantes diante da caracterização Sócio demográfica da amostra transversal quanto ao sexo, idade, tempo de formação e tempo de atuação na Instituição de Saúde. Rio de Janeiro, RJ, Brasil, 2014.

\begin{tabular}{|c|c|c|c|c|c|c|c|c|}
\hline Variáveis & Categoria & Média & DP & $\begin{array}{c}\text { Valor } \\
\text { Mínimo }\end{array}$ & $\begin{array}{c}\text { Valor } \\
\text { Máximo }\end{array}$ & Mediana & $\mathbf{N}$ & $\%$ \\
\hline \multirow{2}{*}{ Sexo } & Feminino & & & & & & 71 & 69,6 \\
\hline & Masculino & & & & & & 31 & 30,4 \\
\hline \multicolumn{9}{|l|}{ Idade } \\
\hline & 29 & & & & & & 22 & 21,5 \\
\hline & $30 \quad 39$ & & & & & & 60 & 58,8 \\
\hline & $40 \quad 49$ & & & & & & 13 & 12,7 \\
\hline & $50 \quad 59$ & & & & & & 6 & 6,0 \\
\hline & $\geq 60$ & & & & & & 1 & 1,0 \\
\hline TOTAL & & 35,2 & 7,7 & 23 & 61 & 33 & 102 & 100 \\
\hline \multicolumn{9}{|l|}{ Tempo de } \\
\hline \multicolumn{9}{|l|}{ Formação } \\
\hline & 02 & & & & & & 55 & 54,0 \\
\hline & 10 & & & & & & 30 & 29,4 \\
\hline & 15 & & & & & & 3 & 2,9 \\
\hline & 16 & & & & & & 5 & 4,9 \\
\hline & 25 & & & & & & 1 & 1,0 \\
\hline & 30 & & & & & & 7 & 6,8 \\
\hline & 33 & & & & & & 1 & 1,0 \\
\hline TOTAL & & 7,9 & 7,5 & 2 & 33 & 5 & 102 & 100 \\
\hline \multicolumn{9}{|c|}{ Tempo de atuação } \\
\hline & 05 & & & & & & 83 & 81,4 \\
\hline & 06 & & & & & & 5 & 4,9 \\
\hline & 11 & & & & & & 6 & 5,9 \\
\hline & 16 & & & & & & 5 & 4,9 \\
\hline & 21 & & & & & & 3 & 2,9 \\
\hline TOTAL & & 4,4 & 5,5 & 1 & 25 & 2 & 102 & 100 \\
\hline
\end{tabular}


TABELA 2 - Distribuição dos participantes diante da caracterização Sócio demográfica da amostra. Rio de Janeiro, RJ, Brasil, 2014.

\begin{tabular}{|c|c|c|c|}
\hline Variáveis & Categorias & $\mathrm{n}$ & $\%$ \\
\hline \multirow[t]{2}{*}{ Sexo } & Feminino & 71 & 69,6 \\
\hline & Masculino & 31 & 30,4 \\
\hline \multirow[t]{16}{*}{ Setor } & Clinica médica & 15 & 14,7 \\
\hline & Centro cirúrgico & 14 & 13,7 \\
\hline & Clinica cirúrgica & 13 & 12,8 \\
\hline & Pronto socorro adulto & 13 & 12,8 \\
\hline & UTI adulto & 11 & 10,8 \\
\hline & Unidade de Transplante & 7 & 6,9 \\
\hline & Clinica cirúrgica neurológica & 5 & 4,9 \\
\hline & Neurologia & 5 & 4,9 \\
\hline & Clinica cirúrgica ortopedia & 4 & 3,9 \\
\hline & Unidade de Cardiologia & 4 & 3,9 \\
\hline & Urologia & 3 & 2,9 \\
\hline & Não respondeu & 3 & 2,9 \\
\hline & Radiologia & 2 & 2,0 \\
\hline & Educação continuada & 1 & 1,0 \\
\hline & Comissão de Curativo & 1 & 1,0 \\
\hline & Ginecologia & 1 & 1,0 \\
\hline \multirow[t]{2}{*}{ Participa atividades de pesquisa graduação } & Não & 70 & 68,6 \\
\hline & $\operatorname{Sim}$ & 32 & 31,4 \\
\hline \multirow[t]{2}{*}{ Lato-sensu } & $\operatorname{Sim}$ & 52 & 51,5 \\
\hline & Não & 49 & 48,5 \\
\hline \multirow[t]{15}{*}{ Qual lato-sensu } & $\mathrm{CCIH}$ & 5 & 4,9 \\
\hline & Não possui & 50 & 49,0 \\
\hline & Uti adulto & 12 & 11,8 \\
\hline & Enfermagem do trabalho & 10 & 9,8 \\
\hline & Urgência e emergência & 4 & 3,9 \\
\hline & Saúde pública & 3 & 2,9 \\
\hline & Nefrologia & 3 & 2,9 \\
\hline & Não respondeu & 3 & 2,9 \\
\hline & Saúde da mulher & 2 & 2,0 \\
\hline & UTI neonato & 2 & 2,0 \\
\hline & Residência em clínica cirúrgica & 2 & 2,0 \\
\hline & Dermatologia & 1 & 1,0 \\
\hline & Estomoterapia & 1 & 1,0 \\
\hline & Médico-cirúrgica & 1 & 1,0 \\
\hline & Oncologia & 1 & 1,0 \\
\hline \multirow[t]{2}{*}{ Stricto-sensu } & Não & 98 & 96,1 \\
\hline & Sim & 4 & 3,9 \\
\hline \multirow[t]{5}{*}{ Participa de Encontros/ Jornadas } & Mensalmente & 62 & 60,8 \\
\hline & Nunca & 17 & 16,7 \\
\hline & Anualmente & 13 & 12,8 \\
\hline & Semestralmente & 5 & 4,9 \\
\hline & Não respondeu & 5 & 4,9 \\
\hline \multirow[t]{2}{*}{ Participa de Comissão Estudo } & Semestralmente & 69 & 67,7 \\
\hline & Quinzenalmente & 15 & 14,7 \\
\hline
\end{tabular}




\begin{tabular}{|c|c|c|c|}
\hline & Nunca & 10 & 9,8 \\
\hline & Anualmente & 2 & 2,0 \\
\hline & Mensalmente & 2 & 2,0 \\
\hline & Semanalmente & 2 & 2,0 \\
\hline & Não respondeu & 2 & 2,0 \\
\hline \multirow[t]{7}{*}{ Participa de Atividades Educacionais } & Quinzenalmente & 39 & 38,2 \\
\hline & Nunca & 27 & 26,5 \\
\hline & Semestralmente & 17 & 16,7 \\
\hline & Semanalmente & 10 & 9,8 \\
\hline & Anualmente & 5 & 4,9 \\
\hline & Mensalmente & 2 & 2,0 \\
\hline & Não respondeu & 2 & 2,0 \\
\hline \multirow[t]{7}{*}{ Acesso a Revistas científicas } & Quinzenalmente & 33 & 32,4 \\
\hline & Nunca & 26 & 25,5 \\
\hline & Semanalmente & 22 & 21,6 \\
\hline & Anualmente & 8 & 7,8 \\
\hline & Semestralmente & 5 & 4,9 \\
\hline & Mensalmente & 4 & 3,9 \\
\hline & Não respondeu & 4 & 3,9 \\
\hline \multirow[t]{6}{*}{ Internet pesquisa } & Diariamente & 79 & 77,5 \\
\hline & Semanalmente & 10 & 9,8 \\
\hline & Mensalmente & 4 & 3,9 \\
\hline & Quinzenalmente & 6 & 5,9 \\
\hline & Semestralmente & 2 & 2,0 \\
\hline & Não respondeu & 1 & 1,0 \\
\hline \multirow[t]{3}{*}{ Recebeu treinamento } & Sim & 46 & 54,8 \\
\hline & Não & 38 & 45,2 \\
\hline & & 102 & 100 \\
\hline
\end{tabular}

Observa-se que a participação em pesquisa durante a graduação não é muito efetiva, mas a busca por uma qualificação profissional em nível de especialização é superior, correspondendo a $51 \%$ dos participantes que realizaram alguma especialização, enquanto $49 \%$ não.

A tabela 3 expõe a análise das questões sobre prevenção, avaliação e classificação de UPP com $90 \%, 80 \%$ e $70 \%$ de acertos.

$\mathrm{Na}$ análise da Tabela 3 sobre o índice de acertos de $90 \%$, $80 \%$ e $70 \%$ sobre Avaliação e Classificação de UPP, as respostas com $90 \%$ de acertos obteve-se um total de 3/102 (2,94\%) e para Prevenção 5/I02 (4,90\%), equivalendo para as respostas corretas abaixo de $90 \%$ sobre Avaliação e Classificação de UPP 99/I02 (97,06\%) e para Prevenção 97/I02 (95, 10\%).

Para as respostas com $80 \%$ sobre Avaliação e Classificação de UPP, obteve-se 8/102 (7,84\%) e para Prevenção 18/102 (17,65\%) e para as respostas com 70\% sobre Avaliação e Classificação de UPP, obteve-se 32/102 (31,37\%) e para Prevenção, 33/102 (32,35\%). Para as respostas corretas inferiores a $70 \%$ sobre Avaliação e Classificação de UPP, obteve-se 70/ 102 (68,63\%) e para Prevenção, $69 / 102(67,65)$.
A tabela 4 representa a incidência de acertos das questões sobre Prevenção de UPP.

$\mathrm{Na}$ análise da tabela 4 sobre as questões que avaliaram o conhecimento dos enfermeiros sobre prevenção de UPP, observa-se que os enfermeiros apresentaram um percentual inferior a $70 \%$ de acertos nas questões 6,20 , 31 e 33.

A tabela 5 representa a incidência de acertos das questões sobre Avaliação e Classificação de UPP.

$\mathrm{Na}$ análise da Tabela 5 sobre as questões que avaliaram o conhecimento dos enfermeiros sobre avaliação e classificação de UPP, observa-se que os enfermeiros apresentaram um percentual de $99 \%$ a $80 \%$ em 7 questões $(2,19,21,23,24,39$ e 40), de $79 \%$ a $70 \% 8$ questões $(4,10,12,18,30,34,37$ e 41$)$ e com percentual inferior a $70 \%$ de acertos em 18 de 33 questões $(3,5,7,8,11,13$, $14,15,16,17,22,25,26,27,28,29,35$ e 36$)$.

\section{DISCUSSÃO}

Neste estudo, os resultados indicam que o gênero feminino ainda é o de maior constância na profissão devido suas características históricas, mas este quadro está 
TABELA 3 - Prevenção, Avaliação e Classificação de UPP considerando $90 \%, 80 \%$ e $70 \%$ de acertos das respostas. Rio de Janeiro, RJ, Brasil, 2014.

\begin{tabular}{|l|c|c|}
\hline Percentual de acertos & $\begin{array}{c}\text { Avaliação e Classificação } \\
\mathbf{n}(\%)\end{array}$ & $\begin{array}{c}\text { Prevenção } \\
\mathbf{n}(\%)\end{array}$ \\
\hline $\mathbf{9 0 \%}$ de acertos & $3(2,94)$ & $5(4,90)$ \\
\hline menos de $\mathbf{9 0} \%$ de acertos & $99(97,06)$ & $97(95,10)$ \\
\hline $\mathbf{8 0} \%$ de acertos & $8(7,84)$ & $18(17,65)$ \\
\hline menos de $\mathbf{8 0} \%$ de acertos & $94(92,16)$ & $84(82,35)$ \\
\hline $\mathbf{7 0 \%}$ de acertos & $32(31,37)$ & $33(32,35)$ \\
\hline menos de $\mathbf{7 0}$ de acertos & $70(68,63)$ & $69(67,65)$ \\
\hline
\end{tabular}

TABELA 4 - Incidência de acertos e erros das questões sobre Prevenção de UPP.

Rio de Janeiro, RJ, Brasil, 2014.

\begin{tabular}{c|l|c|c}
\multirow{2}{*}{ Item } & \multicolumn{1}{|c|}{ Questão } & \multicolumn{2}{|c}{ Corretas } \\
\cline { 3 - 4 } & \multicolumn{1}{|c}{$\mathbf{n}$} & \multicolumn{1}{|c}{$(\%)$} \\
\hline 1 & O estágio I da úlcera por pressão é definido como sendo um eritema que não embranquece. (V) & 84 & $82,35 \%$ \\
\hline 32 & Uma cicatriz de úlcera por pressão poderá lesar mais rapidamente que a pele íntegra. (V) & 79 & $77,40 \%$ \\
\hline 38 & $\begin{array}{l}\text { As úlceras por pressão de estágio II podem ser extremamente doloridas pela exposição das } \\
\text { terminações nervosas. (V) }\end{array}$ & 72 & $70,60 \%$ \\
\hline 9 & $\begin{array}{l}\text { Úlcera por pressão no estágio IV apresentam uma perda de pele total com intensa destruição e } \\
\text { necrose tissular ou danos aos músculos, ossos ou estruturas de suporte. (V) }\end{array}$ & 72 & $70,60 \%$ \\
\hline 6 & Uma úlcera por pressão em estágio III é uma perda parcial de pele envolvendo a epiderme. (F) & 59 & $57,80 \%$ \\
\hline 31 & As úlceras por pressão são feridas estéreis. (F) & 42 & $41,10 \%$ \\
\hline 20 & Úlcera por pressão no estagio II apresentam uma perda de pele na sua espessura total. (V) & 61 & $59,80 \%$ \\
\hline 33 & Uma bolha no calcâneo não deve não deve ser motivo de preocupação. (F) & 37 & $36,30 \%$ \\
\hline
\end{tabular}

TABELA 5 - Incidência de acertos e erros das questões sobre Avaliação e Classificação de UPP. Rio de Janeiro, RJ, Brasil, 2014.

\begin{tabular}{|c|c|c|c|}
\hline \multirow{2}{*}{ Itens } & \multirow{2}{*}{ Questão } & \multicolumn{2}{|c|}{ Corretas } \\
\hline & & $n=102$ & $100 \%$ \\
\hline 2 & $\begin{array}{l}\text { São fatores e risco para o desenvolvimento de úlcera por pressão: mobilidade, incontinência, nutrição } \\
\text { inadequada e alteração do nível de consciência. }(V)\end{array}$ & 99 & $97 \%$ \\
\hline 21 & A pele deve permanecer limpa e seca. (V) & 99 & $97 \%$ \\
\hline 23 & Lençóis móveis ou forros devem ser utilizados para transferir ou movimentar pacientes. (V) & 98 & $96 \%$ \\
\hline 40 & Programas educacionais podem reduzir a incidência de úlcera por pressão. (V) & 95 & $93,10 \%$ \\
\hline 39 & $\begin{array}{l}\text { Para as pessoas que tem incontinência urinaria, a limpeza da pele deve ocorrer no momento que se } \\
\text { sujar e nos intervalos de rotina. (V) }\end{array}$ & 94 & $92,10 \%$ \\
\hline 19 & As pessoas que permanecem na cadeira devem ter uma almofada para proteção. (V) & 89 & $87,20 \%$ \\
\hline 24 & $\begin{array}{l}\text { A mobilização e a transferência de pacientes totalmente dependentes devem ser feitas por duas ou } \\
\text { mais pessoas. }(V)\end{array}$ & 82 & $80,40 \%$ \\
\hline 41 & $\begin{array}{l}\text { Pacientes hospitalizados precisam ser avaliados quanto ao risco para úlcera por pressão uma única } \\
\text { vez. (F) }\end{array}$ & 79 & $77,40 \%$ \\
\hline 12 & Uma escala com horários para mudança de decúbito deveria ser escrita para cada paciente. (V) & 77 & $74,50 \%$ \\
\hline 10 & Uma ingestão dietética adequada de proteínas e calorias deve ser mantida durante a doença. (V) & 75 & $73,50 \%$ \\
\hline 37 & A fricção pode ocorrer ao movimentar a pessoa para cima na cama. (V) & 75 & $73,50 \%$ \\
\hline 18 & $\begin{array}{l}\text { As pessoas que podem aprender devem ser orientadas a mudar seu peso a cada } 15 \text { minutos } \\
\text { enquanto sentadas na cadeira. (V) }\end{array}$ & 74 & $72,50 \%$ \\
\hline 30 & A pele macerada pela umidade danifica-se facilmente. (V) & 74 & $72,50 \%$ \\
\hline 4 & Água quente e sabonete podem ressecar a pele e aumentar o risco para úlcera por pressão. (V) & 73 & $71,50 \%$ \\
\hline 34 & Uma boa maneira de diminuir a pressão nos calcâneos é elevá-los do leito. (V) & 73 & $71,50 \%$ \\
\hline
\end{tabular}




\begin{tabular}{|c|c|c|c|}
\hline 29 & $\begin{array}{l}\text { Toda pessoa avaliada como em risco para desenvolver úlcera por pressão, deveria ser colocada em } \\
\text { colchão redutor de pressão (Ex.: colchão d'água). (V) }\end{array}$ & 67 & $65,70 \%$ \\
\hline 36 & Cisalhamento é a força que ocorre quando a pele adere a uma superfície e o corpo desliza. (V) & 67 & $65,70 \%$ \\
\hline 27 & $\begin{array}{l}\text { Pacientes e familiares devem ser orientados quanto as causas e fatores de risco para o } \\
\text { desenvolvimento de úlcera por pressão. (V) }\end{array}$ & 66 & $64,70 \%$ \\
\hline 16 & $\begin{array}{l}\text { A cabeceira da cama deve ser mantida em um baixo grau de elevação (de preferencia, não maior que } \\
\text { um ângulo de } 30 \text { graus) consistente com as condições médicas. }(V)\end{array}$ & 61 & $59,80 \%$ \\
\hline 26 & $\begin{array}{l}\text { Todo paciente admitido na Unidade de Terapia Intensiva deve ser submetido a avaliação do risco para } \\
\text { o desenvolvimento de úlcera por pressão. (V) }\end{array}$ & 61 & $59,80 \%$ \\
\hline 7 & $\begin{array}{l}\text { Todos os indivíduos devem ser avaliados na admissão no hospital quanto ao risco para desenvolver } \\
\text { úlcera por pressão. (V) }\end{array}$ & 60 & $58,80 \%$ \\
\hline 15 & Na posição lateral, a pessoa deve ficar em ângulo de 30 graus com a cama. (V) & 59 & $57,80 \%$ \\
\hline 25 & A reabilitação deve ser instituída, se o estado geral do paciente permitir. (V) & 58 & $56,80 \%$ \\
\hline 5 & É importante massagear as proeminências ósseas se estiverem hiperemiadas.(F) & 47 & $46,10 \%$ \\
\hline 8 & $\begin{array}{l}\text { Amido de milho, cremes e curativos transparentes (tipo Tegaderm } \circledast \text { ou Opsite } \circledast \text { ) e curativos de } \\
\text { hidrocolóides (tipo Duoderm } ₫ \text { ) não protegem contra os efeitos da fricção. }(V)\end{array}$ & 46 & $45,10 \%$ \\
\hline 35 & $\begin{array}{l}\text { Todo cuidado administrado para prevenir ou tratar úlceras por pressão não necessita ser } \\
\text { documentado. }(F)\end{array}$ & 46 & $45,10 \%$ \\
\hline 28 & As proeminências ósseas podem ficar em contato direto uma com a outra. (F) & 39 & $38,20 \%$ \\
\hline 14 & Rodas d'água ou almofadas tipo argola auxiliam na prevenção de úlcera por pressão. (F) & 32 & $31,40 \%$ \\
\hline 11 & As pessoas que só ficam no leito devem ser reposicionadas a cada 3 horas. (F) & 31 & $30,40 \%$ \\
\hline 13 & Protetores de calcâneos como luvas d'água aliviam a pressão nos calcâneos. (F) & 29 & $28,40 \%$ \\
\hline 22 & $\begin{array}{l}\text { Medidas de prevenção não necessitam ser usadas para prevenir novas lesões quando o paciente já } \\
\text { possui úlcera por pressão. }(F)\end{array}$ & 24 & $23,50 \%$ \\
\hline 17 & $\begin{array}{l}\text { Uma pessoa que não pode se movimentar deve ser reposicionada, enquanto sentada na cadeira a } \\
\text { cada } 2 \text { horas. (F) }\end{array}$ & 17 & $16,70 \%$ \\
\hline 3 & $\begin{array}{l}\text { Todos os indivíduos de risco para úlcera por pressão devem ter uma inspeção sistemática da pele } \\
\text { pelo menos uma vez por semana. }(F)\end{array}$ & 13 & $12,70 \%$ \\
\hline
\end{tabular}

mudando, pois quando se faz um comparativo entre os gêneros em anos anteriores, observa-se que atualmente "o aumento de homens na profissão é gradual e estável, o que se deve, sobretudo, à segurança, estabilidade e garantias de postos de trabalho que a área oferece" (13).

O estudo realizado por Pieper \& Mott ${ }^{13}$ que originou o instrumento deste estudo "PUKT" com 75 enfermeiros de cuidados intensivos constatou que em 19 (57,6\%) dos itens do questionário original, houve mais do que $90 \%$ de acertos, sendo que $3(9,1 \%)$ itens ficaram entre 70 e $89,9 \%$ de acertos, 4 (12,I\%) itens ficaram entre 50 e $69,9 \%$ de acertos e 7 (2I,2 \%) itens a porcentagem de acertos foi inferior a $50 \%$.

Quando comparamos os resultados deste estudo com o de Pieper \& Mott ${ }^{(13)}$, na avaliação de acerto de $90 \%$ dos itens, observamos que o índice de acerto entre os enfermeiros cariocas é inferior ao americano.

Em um estudo brasileiro realizado com 386 profissionais de enfermagem ${ }^{(14)}$, sendo composto por $136(35,2 \%)$ enfermeiros e $250(64,8 \%)$ auxiliares/técnicos de enfermagem, que atuam diretamente na assistência a pacientes adultos e idosos em um hospital universitário, o qual se aplicou o PUKT adaptado, a porcentagem média de acertos no teste de conhecimento foi de $79,4 \%$ para os enfermeiros, indicando falhas no conhecimento.
Em recente estudo transversal realizado com 49 enfermeiros de um hospital municipal também do estado do Rio de Janeiro, os pesquisadores também identificaram um déficit no conhecimento dos profissionais analisados, semelhante aos resultados deste estudo. Os autores referem que $67 \%$ dos avaliados não realizam análise de risco de formação de úlceras e nem utilizam métodos de prevenção ${ }^{15}$.

Iranmanesh, Rafiei e Foroogh Ameri(16) desenvolveram no Irã outro estudo utilizando o "PUKT" para determinar o conhecimento de 126 enfermeiros iranianos que atuam em unidades de cuidados críticos sobre LPP. O estudo mostrou que o nível de conhecimento dos enfermeiros sobre LPP foi insuficiente, sugerindo a necessidade de uma educação mais ativa destes profissionais. $\mathrm{Na}$ avaliação dos dados, percebeu-se que o conhecimento dos enfermeiros sobre prevenção de LPP foi mais alto que o reconhecimento quanto a classificação e avaliação, este achado coincide com os dados deste estudo, indicando que em ambos os países em desenvolvimento há falhas no nível de conhecimento dos profissionais, principalmente quanto a avaliação das LPPs.

Em contrapartida, um recente estudo transversal realizado na Austrália com 998 enfermeiros demonstrou uma taxa de mais de $78,6 \%$ dos participantes com mais de $70 \%$ de acer- 
tos $^{(17)}$.A comparação dos resultados nos países desenvolvidos e em desenvolvimento demonstra a diferença na qualidade de preparo dos profissionais envolvidos na assistência.

A falta de adesão dos profissionais que atuam direta e indiretamente com os pacientes ainda é alta. Nota-se como determinantes para esta situação a falta de conhecimento e de empenho, poucos recursos empregados no treinamento e à escolha inadequada de dispositivos para prevenção e tratamento. Quando se compara os custos utilizados para o treinamento das equipes com os custos utilizados no tratamento, independente da classificação da LPP confirma-se que o primeiro tem um custo significativamente menor ${ }^{(18)}$.

Por fim, espera-se que os resultados deste estudo possam auxiliar as equipes gestoras no desenvolvimento de treinamentos educacionais mais efetivos junto a suas equipes, favorecendo o conhecimento mais ativo dos seus profissionais e consequentemente reduzindo os problemas que a LPP ocasiona ao paciente/família/sociedade.

\section{CONCLUSÃO}

Este estudo indica que os enfermeiros mostraram conhecimento insuficiente sobre a classificação, avaliação e prevenção de úlceras por pressão, embora conhecessem mais sobre a prevenção do que sobre a avaliação e classificação. Em futuros estudos, seria interessante a aplicação de metodologias ativas de capacitação para estes profissionais, seguida por uma avaliação do impacto destes cursos no conhecimento destes indivíduos.

$O$ enfermeiro, por ser um profissional que precisa impor medidas que favoreçam a redução das taxas de incidência e prevalência de LPP, necessita ser e estar capacitado para tal realidade, e para tal sugere-se que medidas educativas institucionais sejam implantadas de forma mais efetiva o que ocasionaria uma redução significativa na qualidade da prevenção de riscos do surgimento da LPP, refletindo também na redução dos custos totais referentes a internação e tratamento dos pacientes. 


\section{REFERÊNCIAS}

I. Saghaleini SH, Dehghan K, Shadvar K, Sanaie S, Mahmoodpoor A, Ostadi Z. Pressure ulcer and nutrition. Indian J Crit Care Med. [Internet]. 2018 [acesso em 05 set 2018]; Apr;22(4):283-289. Disponível em https://www.ncbi.nlm. nih.gov/pmc/articles/PMC5930532/

2. Dreyfus J, Gayle J, Trueman P, Delhougne G, Siddiqui A.Bliss M, Simini B. Assessment of Risk Factors Associated With Hospital-Acquired Pressure Injuries and Impact on Health Care Utilization and Cost Outcomes in US Hospitals. Am J Med Qual.[Internet]. 2018 [acesso em 10 ago 2018]; Jul;33(4):348-358. Disponível em https://doi. org/ I0.1 I77// 0628606/774674 I

3. Catania KHC, James P, Madison M, Moran M, Ohr M.Wound wise: PUPPI: the pressure ulcer prevention protocol interventions. [Internet].Am J Nurs. [Internet] 2007 [acesso em 5 maio 2017]; 107(4):44-52. Disponível em https://europepmc.org/abstract/med/I74I 3732

4. European Pressure Ulcer Advisory Panel and National Pressure Ulcer Advisory Panel. Prevention and treatment of pressure ulcers: quick reference guide. Washington DC: National Pressure Ulcer Advisory Panel; 2009. Disponível em: www.epuap.org. Acessado em maio de 2012.

5. Ziraldo C, Solovyev A, Allegretti A, Henzel MK, Mi Q, Vodovotz Y.A Computational,Tissue-Realistic Model of Pressure Ulcer Formation in Individuals with Spinal Cord Injury. Plos comput Biol. [Internet]. 2015 [acesso em 30 jul 2017]; Jun; I I(6): el004309. Disponível em https://www.ncbi.nlm.nih. gov/pmc/articles/PMC4482429/

6. Vangilder C, Amlung S, Harrison P, Meyer S. Results of the 2008-2009 International Pressure Ulcer Prevalence Survey and a 3-year, acute care, unit-specific analysis. Ostomy Wound Manage. [ Internet]. 2009 [ acesso em I set 2018]; Nov I;55(I I):39-45. Disponível em https://www.o-wm. com/content/results-2008-\%E2\%80\%93-2009-international-pressure-ulcer-prevalence\%E2\%84\%A2-survey-and-a3-year-acute-care-

7. Hospital Universitario "Marqués de Valdecilla". Dirección de Enfermería. Área de Formación y Calidad. Manual de Prevención y Tratamiento de las Úlceras por Presión. Documento reconocido de interés profesional por el GNEAUPP (Grupo Nacional para el Estudio y Asesoramiento en Úlceras por Presión y Heridas Crónicas). Espanha, 2003.

8. Caliri MHL, Pieper B, Cardozo LJ. "Development of Distance Learning Modules about Chronic Wound Prevention and Treatment in Brazil”, 200I-2002. Disponível em:http://www2.eerp.usp.br/site/grupos/feridascronicas/ index.php?option=com_content\&view=article\&id= I 8\&Itemid=27.

9. Dreyfus J, Gayle J, Trueman P, Delhougne G, Siddiqui A. As- sessment of Risk Factors Associated With Hospital-Acquired Pressure Injuries and Impact on Health Care Utilization and Cost Outcomes in US Hospitals. Am J Med Qual. [Internet]. 2018 [acesso em 5 set 20I8]; Jul;33(4):348-358. Disponível em https://doi.org/I0.I I77/I0628606 I774674 I

10. Galvão NS, Serique MA, Santos VL, Nogueira PC. Knowledge of the nursing team on pressure ulcer prevention. Rev Bras Enf. [Internet]. 2017 [acesso em 2 mai 20I8];Apr;70(2):294300. Disponível em http://www.scielo.br/scielo.php?script=sci_arttext\&pid=S0034-7 I 6720 I 7000200294

I I. Fernandes LM, Caliri MHL, Haas VJ. The effect of educative interventions on the pressure ulcer prevention knowledge of nursing professionals. Acta Paulista de Enfermagem. [Internet]. 2008 [ acesso em 5 set 20I8]; 2I(2):305-3II. Disponível em http://www.scielo.br/scielo.php?script=sci_arttext\&pid=SO I03-2 1002008000200012

12. Piepper B, Mott M. Nurse's Knowledge of Pressure Ulcer Prevention, stading and description. Advances in Wound Care. [Internet]. 1995 [acesso em 10 mai 2018]; 8(3): 34-48. Disponível em https://www.ncbi.nlm.nih.gov/pubmed/7795877

13. Chianca TCM; Rezende JFP, Borges EL, Nogueira VL, Caliri MHL. Pressure ulcer knowledge among nurses in a Brazilian university hospital. Ostomy Wound Management. [Internet]. 2010 [acesso em 5 set 2018]; 56(10):58-64. Disponível em https://www.o-wm.com/content/pressure-ulcer -knowledge-among-nurses-brazilian-university-hospital

14. Oliveira BG, Freitag VL, Victorino VZ. O conhecimento dos enfermeiros sobre o cuidado da lesão por pressão. Rev Enfemagem Atual. [Internet]. 2018 [acesso em 5 set 20l8]; Jan/Fev/Mar 84(22): 59-68. Disponível em https://revistaenfermagematual.com.br/uploads/revistas/22/05.pdf

15. Iranmanesh S, Hossein R, and Golnaz FA. Critical care nurses' knowledge about pressure ulcer in southeast of Iran. International wound journal. [Internet]. 201 I [acesso em 15 mar 2018]; Oct;8(5):459-64. Disponível em https://doi. org/I0.1 I I I/j.1742-48IX.20II.008I7.x

16. Barakat-Johnson M, Barnett C, Wand T, White K. Knowledge and Attitudes of Nurses Toward Pressure Injury Prevention: A Cross-Sectional Multisite Study. J Wound Ostomy Continence Nurs. [Internet]. 2018 [acesso em 22 abr 20I8]; May/Jun;45(3):233-237. Disponível em https:// insights.ovid.com/pubmed?pmid=29722753

17. Demarré L, Van Lancker A, Van Hecke A, Verhaeghe S, Grypdonck M, Lemey J, Annemans L, Beeckman D. The cost of prevention and treatment of pressure ulcers: a systematic review. Int J Nurs Stud. [Internet]. 2015 [acesso em $20 \mathrm{abr}$ 2018]; Nov;52(I I):1754-74. Disponível em https://www.sciencedirect.com/science/article/pii/ S00207489/500200X?via\%3Dihub. 